\title{
Mehr als eine Mathematikstunde: Platons Menon
}

\section{$\boldsymbol{Z f E}$}

\author{
Patrick Bühler
}

Zusammenfassung: Seit mindestens zwei Jahrhunderten besteht in der Pädagogik eine Fixierung auf die in Platons Menon enthaltene ,Mathematiklektion', die immer wieder als ein Meisterstück der sokratischen Methode gepriesen wird. Indem man jedoch allein der ,Mathematikstunde' Beachtung schenkt, bleibt das negative Ende des Menon ausgespart. Überdies wird die ,Unterrichtsstunde' häufig als Beispiel einer positiven sokratischen Hebammenkunst angeführt. Auf diese Weise gerät eine weitere mögliche medizinische, therapeutische" Verbindung in Vergessenheit, die zwischen einer Hebamme, mit der Sokrates sich selbst in Platons Theätet vergleicht, und einem Zitterrochen besteht, mit dem er im Menon gleichgesetzt wird.

Schlüsselwörter: Sokrates $\cdot$ Sokratische Methode $\cdot$ Mäeutik $\cdot$ Menon ·

Geschichte der Pädagogik

\section{More than a maths lesson: Plato's Meno}

\begin{abstract}
For the past two hundred years in German educational science the 'maths lesson' of Plato's Meno has received attention - always to be celebrated as a masterpiece of Socratic teaching. Yet, by taking only the 'maths lesson' into account the negative ending of Meno is overlooked. Thus, because generally the 'maths lesson' is used as an example for a positive Socratic maieutic teaching method, a second possible medical, 'therapeutic' link between a midwife, with which Socrates compares himself in Plato's Theaetetus, and a torpedo fish, with which he equates himself in Meno, is usually overlooked.
\end{abstract}

Keywords: Socrates $\cdot$ Socratic method $\cdot$ Meno $\cdot$ Maieutics $\cdot$ History of education

\footnotetext{
Online publiziert: 24.07 .2010

(C) VS Verlag für Sozialwissenschaften 2010

Dr. P. Bühler $(\bowtie)$

Institut für Erziehungswissenschaft, Universität Bern,

Muesmattstraße 27, 3012 Bern, Schweiz

E-Mail: buehler@edu.unibe.ch
} 
„Man kann wohl den Sokrates nicht im eigentlichen Verstande für einen Edukator der Jugend nach unsern heutigen Begriffen ansehen."

Friedrich Rudolph Walther (1780, S. 21)

\section{Torpedos und Zitterrochen}

Platons Theätet zufolge besteht der ,wichtigste Teil“ von Sokrates’ Aufgaben als Hebamme in seiner unter Umständen , abortiven', negativen Didaktik: Der Philosoph selbst gebäre nichts, sondern prüfe nur, „ob der Geist des Jünglings eine Schein- und Lügengeburt zutage bringt, oder etwas Echtes und Wahres“" (Platon 2004c, S. 42/150a-c). So diagnostiziert Sokrates am Ende des Dialogs denn auch bei seinem Gesprächspartner Theätet nichts als „Windgeburten“, ,,die nicht des Aufziehens wert“ seien (Platon 2004c, S. 144/210b):

Solltest du also weiterhin, mein Theätet, in Versuchung kommen wieder schwanger $\mathrm{zu}$ werden und wenn du es wirklich wirst, so wird die jetzige Untersuchung zur Folge haben, dass es dann bessere Gedanken sind, die dich erfüllen; solltest du aber leer bleiben, so wirst du deiner Umgebung weniger lästig sein und milder, indem du bescheidentlich dich des Glaubens entschlägst zu wissen, was du nicht weißt. Denn nur so viel vermag meine Kunst, aber nicht mehr (Platon 2004c, S. 144/210b-c).

Die Wirkung dieser negativen „Kunst“ vergleicht Menon in Platons gleichnamigem Dialog mit der eines „Zauberer[s]“, der ihn ,behext“ (m, S. 36/80a) und der in einer fremden Stadt wohl verhaftet würde (vgl. m, S.37/80b). ${ }^{1}$ So ist Menon, nachdem seine Versuche zu bestimmen, was Tugend ist, drei Mal an Sokrates' Einwänden gescheitert sind, gänzlich verwirrt: Sokrates bringe ihn ,,völlig in [s]eine Gewalt“, er wisse „,nicht mehr aus und ein“ (m, S. 36/80a). Schon vor seiner Unterredung mit Sokrates hat Menon gehört, dass dessen ,ganzes Tun und Treiben darauf hinausläuft, selbst wie in der Irre zu gehen und die anderen an sich irre zu machen“ (m, S.36/79d-80a). Deswegen vergleicht Menon den Philosophen auch im „Scherze“ mit einem Zitterrochen:

Denn auch dieser macht jeden, der ihm nahekommt und ihn berührt, erstarren. Ewas derart hast auch du denn, wie mich dünkt, jetzt mir angetan. Du hast mich erstarren gemacht. Denn tatsächlich bin ich starr an Seele und Mund und weiß nicht, was ich antworten soll (m, S. 36 f./80a-b).

Der Vergleich zwischen Sokrates und einem Zitterrochen führt weiter, als man auf Anhieb vielleicht annehmen könnte. Von der Familie dieser Fische, der torpedinidae, sind heute 35 Arten bekannt, ihre ,an den Seiten des Kopfes und des Vorderkörpers“" gelegenen elektrischen Organe erzeugen Spannungen von ,,70 bis 300 V“([Brockhaus] 2001): ,Schläge“ von einer solchen Stärke reichen unstreitig aus, ,dem Menschen äußerst empfindlich zu sein und die verlangte Beute zu tödten oder zu betäuben“ (Keserstein 1859, S. 34). Die durch solche Stromstöße hervorgerufene Lähmung bestimmt nicht nur den Vergleich, den Sokrates' Gesprächspartner anstellt, sondern auch schon die Bezeichnung des Fisches selbst: Im Griechischen bedeutet nárkē sowohl „Erstarrung“, „Krampf ‘ und ,Lähmung“ als auch ,Zitterrochen'. Und auch im Lateinischen meint torpedo ebenso ,Lähmung“, 
,Stumpfheit‘ und ,Dumpfheit‘ wie den Fisch. So wird das Tier z. B. auch in Friedrich Justin Bertuchs Bilderbuch für Kinder (1790) als „Zitter-Roche, oder der Torpedo“ aufgeführt, der „beynahe“ so aussehe „wie ein runder Teller mit einem Schwanze“: „Er lebt von Fischen, und betäubt die kleinern, [die] so über ihn wegschwimmen, wenn er im Sande liegt, dass sie auf ihn hinfallen, und er sie dann fressen kann“ (Bertuch 1790, $\mathrm{N}^{\circ}$ 55).

Auch im Englischen wird torpedo seit dem 16. Jahrhundert - andere Namen sind cramp-fish, cramp-ray, numb-fish - zur Bezeichnung des Zitterrochens verwendet (vgl. [OED] 1989, S. 270). Als Terminus für Kriegsgerät kommt der Ausdruck erst 1810 durch die Abhandlung des amerikanischen Malers, Erfinders und Ingenieurs Robert Fulton Torpedo War, and Submarine Explosions in Umlauf: Mit ,torpedo' bezeichnet Fulton verschiedene ,Sorten“ von Seeminen. ${ }^{2}$ Ein halbes Jahrhundert später entstehen die modernen Torpedos: 1866 baut der Engländer Robert Whitehead, der in Fiume - heute Rijeka in Kroatien - arbeitet, den ersten funktionierenden zigarrenförmigen ,submarine locomotive torpedoe[]“ (Gray 1975, S. 26). Innerhalb von zehn Jahren werden die WhiteheadTorpedos zum Verkaufsschlager und ihr Erfinder zum Millionär. Bereits 1877 verfügen die Flotten von 14 Ländern über solche Torpedos. ${ }^{3}$ Mit dem Erfolg der Whitehead-Torpedos gelangt der Begriff auch ins Deutsche: der Ausdruck ist ab 1876 belegt (vgl. Kluge 1975). ${ }^{4}$

Dass der Zitterrochen zwar „die arglos über ihn hinwegschwimmenden Fische“ fängt, „,indem er sie lähmt“, aber dabei „,selbst nicht erstarrt[]“, wird schon in Plinius’ Naturkunde eigens hervorgehoben (Plinius 1979, S. 105/9.143). Und ähnlich ,unnatürlich“ wie ein sich selbst betäubender Zitterrochen wäre ein sich selbst versenkender Torpedo, der folglich auch in Fultons De la machine infernale maritime, ou de la tactique offensive et défensive de la Torpille (1812) - so die französische Übersetzung seines Buches Torpedo War - nirgendwo erwähnt wird. Was in Natur- und Kriegskunde ausgeschlossen scheint, wird nun aber ausgerechnet in der Philosophie reklamiert: Zumindest Sokrates ,torpediert' im Menon im Gegensatz zu Zitterrochen und Torpedos nicht nur sein Gegenüber, sondern ausdrücklich auch sich selbst. Der Philosoph lässt Menons Vergleich nämlich nur dann gelten, wenn der Zitterrochen ,bei seiner starrmachenden Wirkung auf die anderen auch selbst starr“ werde: Wenn Sokrates „die anderen ratlos“ mache, dann sei er ebenfalls „schlechterdings ratlos“ (m, S.37/80 c).

Dass es sich bei Sokrates',Ratlosigkeit“ nicht einfach um einen ,aus didaktischen Gründen gewählten Schein des Nichtwissens“ ([Meyer] 1897, S. 70) handelt, zeigt das ,doppelt" negative Ende des Dialogs. Denn am Schluss des Menon ist nicht nur nach wie vor ungeklärt, was Tugend ist, sondern diese nicht weiter bestimmte Tugend erweist sich auch als nicht lehrbar (vgl. m, S. 72 f./99e-100b). Sören Kierkegaard sieht darin eine Form höherer Ironie. In Über den Begriff der Ironie: Mit ständiger Rücksicht auf Sokrates (1841/1961) hebt er hervor, dass gerade die Behauptung ,Tugend sei nicht lehrbar“ „nicht bloß eine negative Bestimmung, sondern auch eine im ironischen Sinne negative" sei: „Von Lehrbarkeit der Tugend kann so wenig die Rede sein, dass die Tugend vielmehr dermaßen weit hinter dem Individuum zurückliegt, dass zu befürchten ist, sie sei vergessen“ (Kierkegaard 1961, S.61 f.).

In pädagogischen Abhandlungen wird nun meistens nicht dieser ,zweifach“ negative Schluss des Menon angeführt, sondern nur die Passage zitiert, in der Sokrates einem Skla- 
ven Mathematikunterricht erteilt (vgl. m, S. 40 ff./82a-85d). In „,das allgemeine Bewusstsein“ ist allein, so Julius Stenzel 1928 in Platon der Erzieher,

das Mittelstück dieses Dialoges eingegangen, das berühmte „maieutische“ Kunststück des Sokrates, die mathematische Katechese eines jungen Sklaven, aus dem der pythagoreische Lehrsatz für das rechtwinklig-gleichschenklige Dreieck herausgefragt wird (Stenzel 1928, S. 148 f.).

Diese „wahrlich klassische didaktisch-methodische Urszene“ (Terhart 2009, S. 170 f.) wird seit über zweihundert Jahren in der Pädagogik als „,Juwel der Lehrkunst“ und „Muster der sokratischen Lehrart gepriesen“ (Prange 2008, S. 20). Schon 1791 gilt die Stelle als „Meisterstük der Sokratischen Kunst“ (Gräffe 1791, S. 88) und wird, so z.B. Weierstraß (1845), „oftmals als ein schönes Muster der Sokratischen Methode empfohlen“ (Weierstraß 1903, S.316) oder, so Kellner (1862), ,als ein Meisterstück der sokratischen Methode und der Entwicklung gerühmt“" (Kellner 1862, S. 57).

Ein Grund für die anhaltende pädagogische Begeisterung für den Mathematik-Passus ist wohl darin zu suchen, dass er im Gegensatz zum Ende des Dialogs eine ebenso hübsche wie einfache Lösung bereit zu halten scheint. So besteht die ,Mathematiklektion“ des Menon darin, dass der Sklave mithilfe des Philosophen die Diagonale eines ersten Quadrates findet, mit der sich ein zweites konstruieren lässt, das die doppelte Fläche des ersten aufweist. Dabei verhält sich Sokrates vermeintlich didaktisch korrekt: „Damit die Aufgabe auch klar wird, malt Sokrates ein Quadrat in den Sand mit je zwei Fuß Seitenlänge. Didaktisch gesprochen: er gibt dem Gedanken (Quadrat) eine Anschauung, um seine Bedeutung zu sichern“ (Prange 1986, S. 28). Im Gegensatz zu dieser „Aufgabe“ und ihrer Lösung fällt das Ende des Menon didaktisch um einiges zwiespältiger aus. Schon in Christoph Martin Wielands Briefroman Aristipp und einige seiner Zeitgenossen (1800 1802) wird auf die fragwürdigen pädagogischen Konsequenzen des Schlusses hingewiesen, bei dem sich eine nicht näher bestimmte Tugend als nicht lehrbar, sondern allein als „göttliche Schickung“ (m, S. 72/99e) erweist:

Wahrlich, die Behauptung in seinem [Platons] Menon, dass die Tugend keine Frucht des Unterrichts und der Erziehung sein könne, ist nicht sehr geschickt eine bessere Erziehung unsrer immer mehr verwildernden Jugend zu befördern (Wieland 1988, S. 663).

\section{Die ,weite[n] Felder glibbrigen Quarkes“ bei Platon}

Die ,Mathematikstunde“ des Menon wird in der Pädagogik häufig mit den „Hebammenkünsten“ (Hamann 1959, S. 105) des Theätet verknüpft. Tatsächlich lässt sich mit dem Menon, sofern man den Dialog auf die durch den Sklaven gefundene Diagonale reduziert, elegant eine Mäeutik illustrieren, bei der ausschließlich „glücklich gebohren“ (Mosheim 1737, S. 472) wird und von der „Paradoxie des Lernens“ (Stenzel 1928, S. 148) keine Rede mehr ist. So verbindet etwa schon 1760 Moses Mendelssohn in seiner anonym erscheinenden Rezension von Johann Georg Hamanns Sokratischen Denkwürdigkeiten (1759) „Schulweisheit““ und „Hebammenkunst“. Dass „,der Same aller Begriffe, die wir 
erlangen“, in der Seele liege, „so wie die Frucht im Mutterleibe schon die Grundbildung des künftigen Mannes enthält“, beweise Sokrates am „Knaben seines Wirths“ „durch seinen Hebammendienst“: Der Philosoph „lockte durch bloßes Fragen einen tiefsinnigen mathematischen Satz aus dem Munde eines Unwissenden, der kaum wusste, was Länge und Breite sey“ (Mendelssohn 1760, S. 398). Auch im 19. und 20. Jahrhundert werden „Hebammendienst“ und Menon oft vermengt, auch wenn dabei nicht immer der Begriff ,Mäeutik' verwendet wird. ${ }^{5}$ So geht es z.B. nach Johann Christian Friedrich Wetzel im Menon um ,Entwicklung':

Sokratik also knüpft an die schon vorhandenen Begriffe die noch fehlenden an, oder sie entwickelt aus dem schon Bekannten das noch Unbekannte. Auf diese Weise bringt jener große Erfinder und Meister dieser Kunst in dem Platonischen Gespräch Menon durch geschickte Fragen einen Kleinen so weit, dass dieser selbst gleich einem Mathematiker sich sein Viereck ausmisst (Wetzel 1801, S. 6 f.).

Die seit dem 18. Jahrhundert nicht abebbende pädagogische Begeisterung für die ,Mathematikstunde“ des Menon als einer, ,wichtige[n] Darstellung der Sokratischen Manier“ (Gräffe 1791, S. 86) zeigt sich auch daran, dass die Diagonalen-Passage häufig auszugsweise abgedruckt und mit Illustrationen versehen wird. Ein frühes Beispiel dafür findet sich in Johann Friedrich Christoph Gräffes Sokratik: „Um die Verständlichkeit der Sokratischen Fragen zu befördern, zeichne ich hier die Figur bei, von welcher die Rede ist, und setze hinter jede Frage die Benennung der Figur und Linien in Buchstaben“" (Gräffe 1791, S. 88). ${ }^{6}$ Ähnliche Darstellungen kommen - meist mitsamt längeren Ausschnitten aus der ,Mathematikstunde“ - auch im 19. und 20. Jahrhundert in zahlreichen
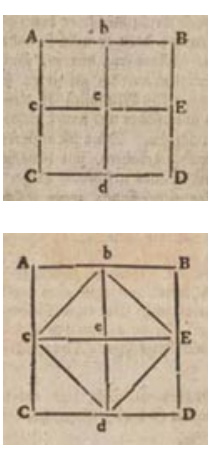
Geschichten der Erziehung und pädagogischen Studien vor. ${ }^{7}$

Beschränkt man sich allein auf die ,Mathematiklektion', die Sokrates dem Sklaven erteilt, dann kann sich die „Erziehung des Verstandes“ (Hamann 1959, S. 106) im Menon natürlich auch nur zwischen dem Philosophen und dem Sklaven abspielen. Betrachtet man hingegen den ganzen Dialog, dann zeigt sich, dass der rechnende Sklave nur als ,Beweis‘ für Sokrates' These ,Lernen ist erinnern“ dient. Wie Johann Jakob Engel in seinem Versuch einer Methode die Vernunftlehre aus Platonischen Dialogen zu entwickeln (1780) resümiert:

Menon fordert davon [dass Lernen Wiedererinnerung sei] einen Beweis; und Sokrates ruft aus dem Gefolge desselben einen jungen Sklaven herbei, den er durch Fragen dahin bringt, dass er einen tiefsinnigen geometrischen Satz von selbst erfinden muss (Engel 1805, S. 13).

Wenn man also vom gesamten Dialog ausgeht, dann handelt es sich bei der ,Mathematikstunde' zwar um ,Schulfunk', aber nicht für den Sklaven, wie die pädagogische Sekundärliteratur in aller Regel suggeriert, sondern für Menon. So kann der Sklave, ohne die leiseste Ahnung von Geometrie zu haben, sich der Diagonale ,erinnern“: Diesen ,Beweis“ benutzt Sokrates, um Menon darzulegen, dass der Sklave ,aus sich selbst das Wissen gewinnt“ (m, S. 47/85d). Deshalb unterbricht der Philosoph die ,Mathematikstunde“ auch zweimal (vgl. m, S. 44 f./84a-d), um ganz didaktisch für Menon deren „Bedeutung zu 
sichern“ (Prange 1986, S. 28): „Du siehst doch, Menon, dass ich ihn [den Sklaven] nichts lehre, sondern alles erfrage?" (m, S. 41/82e)

Aber selbst wenn man nur die ,Mathematiklektion“ betrachtete und Sokrates' Gespräche mit Menon während der ,Stunde“ überginge, müsste ,auffallen, dass der Sklave eigentlich nichts selbst findet, sondern fast immer nur mit Ja oder Nein antwortet, ja dass Sokrates zumeist Alles vorsagt" (Kellner 1862, S. 57). Es liegt eigentlich

auf der Hand, dass man ein solches Verfahren kaum Entwicklung oder Heuristik nennen kann. Was den zu entwickelnden Satz angeht, dass das Quadrat der Diagonale doppelt so groß sei, als das gegebene Quadrat, so dürften die Mathematiker auch manches daran auszusetzen haben. Die Unvollständigkeit der Definitionen fällt zunächst auf; dass in einem Quadrate auch gleiche Winkel sein müssen, ist ganz übergangen worden. Der Ausdruck Fu $\beta$ wird ohne Unterschied als Längen und Flächenmass gebraucht (Kellner 1862, S. 57 f.).

In ,Die sokratische Lehrmethode“ (1849) fragt Otto Schulz deswegen auch, ,ob Diesterweg mit dieser Entwicklung zufrieden sein würde, vorausgesetzt, dass sie nicht im Plato stände, sondern dass ein Lehrer sie bei einer Probelection vorgetragen hätte“? Neben der „Unvollständigkeit der Definitionen“ - Schulz bemängelt ebenfalls, dass das Quadrat gleiche Winkel aufweisen müsse und dass das $\mathrm{Ma} ß$,Fuß als Längen- wie als Flächenmaß verwendet werde - würde der Pädagoge dem Lehrer vorhalten, ,dass er den Schüler eigentlich nichts habe finden lassen, sondern ihm alles haarklein vorgesagt habe, so dass diesem nur übrig blieb, mit Ja oder Nein zu antworten“" (Schulz 1849, S. 641).

Kellners und Schulz' Einwände ließen sich noch weiter zuspitzen. Man könnte sich nämlich fragen, ob denn , die Entscheidungen des Sokrates richtig, ob sein Räsonnement überall bündig und gründlich sei?“ (Engel 1805, S. 21 f.) Verfolgte man diese Spur, so würde man möglicherweise zu Schlüssen gelangen, wie sie Alexander Moszkowski in seiner „respektlose[n] Studie“ Sokrates der Idiot (1917) zieht. Moszkowskis These lautet, dass ,Sokrates nicht nur ein Idiot war, sondern auch, dass Plato ihn als Idioten durchschaute“ (Moszkowski 1917, S.34). Der „Ultrasophismus des Sokrates“, der „Hochgrad des Blödsinns“, sei nur „dank der Magie des Plato“ bisher „den Lesenden nicht zum Bewusstsein“ gekommen (Moszkowski 1917, S. 56, 75). Die Symptome der „Trottelosis“ des Philosophen, die Moszkowski diagnostiziert, sind unter anderem seine „logischen Bocksprünge und spielerischen Wortfälschungen“, der „wahre[] Platzregen von Fehlschlüssen“, die ,gedankliche[n] Karussellfahrt[en]“ sowie die ,weite[n] Felder glibbrigen Quarkes“ der platonischen Dialoge (Moszkowski 1917, S. 31, 49, 67, 107). Dass „Platon nicht ganz selten Fehlschlüsse zu weitgreifenden Folgerungen“ verwende, seine Dialoge ,[s]tarke Proben eines skrupellosen Aus- und Unterlegens“ böten und „etymologische[] Scherze“ aufwiesen, ist auch der Ausgangspunkt von Wilhelm Eckerts Dialektischem Scherz in den frühen Gesprächen Platons (1911). Die „,sophistischen Wendungen und elenktischen Vergewaltigungen“ träten jedoch „,nicht regellos verstreut“ auf, sondern seien auf eine „künstlerische Absicht zurückzuführen“: Platons „Bestreben“ sei es, „Unzureichendes als geradezu unberechtigt, Mehrdeutiges als unsinnig hinzustellen, Inkonsequenzen zu schroffen Widersprüchen zuzuspitzen, kurz, gegebene Aporien zu steigern“" (Eckert 1911, S. 4, 11, 19, 139, 140). ${ }^{8}$ 
In der Rolle eines imaginären Lehrers, der sich direkt an seine imaginäre Zuhörerschaft wendet, hegt Engel große Hoffnungen:

Sie haben Sich, glaube ich, Alle, bei Durchlesung dieses Gesprächs, in dem Fall des Menon oder des jungen Sklaven befunden. Sie haben es überall leicht gemerkt, wo die Wahrheit verfehlt oder getroffen, wo richtig oder wo unrichtig geschlossen ward (Engel 1805, S. 24).

Gleichgültig, wie „leicht“ diese ,Verfehlungen“ wirklich zu erkennen sein mögen, es lässt sich nicht ausschließen, dass Engels Zuhörer sich neben „dem Fall des Menon oder des jungen Sklaven“" außerdem noch ,in dem Fall des Sokrates' befunden haben könnten. So könnte sich Engels imaginäre Schülerschar ja durchaus auch überlegen, wie sie an Sokrates' Stelle fragen und antworten würde. Natürlich wäre es ebenfalls denkbar, dass man „bei Durchlesung“ des Menon sogar auf alle drei ,Fälle“ gleichzeitig achtete, wie es etwa Moszkowski und Eckert machen. Der ,Unterricht` im Menon fände dann nicht mehr zwischen Sokrates und dem Sklaven - wie die pädagogische Sekundärliteratur immer wieder annimmt - oder zwischen Sokrates und Menon statt, sondern ,Lehrer" und „Lektion` wären der Menon selbst, der ,Schüler' aber wäre sein Leser. So kann denn nach Friedrich Schlegel durch den Dialog auch eben gerade nicht „das Selbstdenken“ des Sklaven, sondern bestenfalls das des , überraschte[n] Zuschauer[s]“ angeregt werden. Im „dialogischen Kunstwerke“ sei es nämlich „die gegenseitige Gedankenvermittlung der Redenden, welche selbst de[n] Gegenstand" abgebe (Schlegel 1975, S. 48). Gerade dieses „Unbestimmte“ (Schlegel 1975, S.48) könne, so Schlegel in Lessings Gedanken und Meinungen (1804), „das Selbstdenken“ „erregen“, schließlich sei Philosophie „ein ewiges Suchen und Nichtfinden“" (Schlegel 1975, S. 99):

Wie vortrefflich zum Beispiel sind mehrere Schriften der alten Philosophen, besonders die Platonischen recht eigentlich dazu eingerichtet, das Selbstdenken zu erregen. Ein Widerspruch gegen ein geltendes Vorurteil, oder was irgend sonst die angeborne Trägheit recht kräftig wecken kann, macht den Anfang; dann geht der Faden des Denkens in stetiger Verknüpfung unmerklich fort, bis der überraschte Zuschauer, nachdem jener Faden mit einem Male abreißt, oder sich in sich selbst auflöste, plötzlich vor einem Ziele sich findet, das er gar nicht erwartet hatte; vor sich eine grenzenlose weite Aussicht, und sieht er zurück auf die zurückgelegte Bahn, auf die deutlich vor ihm liegende Windung des Gesprächs, so wird er inne, dass es nur ein Bruchstück war aus einer unendlichen Laufbahn (Schlegel 1975, S. 50).

Einen ähnlichen Standpunkt wie Schlegel nimmt schon rund zweihundert Jahre früher Michel de Montaigne in seinen Essais ein. Platon lege „,seine eigenen verschiedenen und veränderlichen Gedanken“ anderen „,in den Mund“. Diese „Art durch Gespräche zu philosophiren“ gewinne ihre Bedeutung durch die „entgegengesetzte[n] Beweisgründe“, die dargelegt würden, und funktioniere wie ein „Parlament][“ (Montaigne 1992, S. 168 f.). Montaignes und Schlegels Auffassung ist den platonischen Dialogen durchaus angemessen, ist sie doch äußerst ,antik'. So besteht die griechische und römische Philosophie, wie Pierre Hadot in Exercices spirituels et philosophie antique (1993) unterstreicht, nicht aus geschlossenen theoretischen Systemen, sondern aus ,therapeutischen' geistigen Übungen. 
Die platonischen Dialoge seien literarische Muster solcher Übungen (vgl. Hadot 2002, S. 38 ff.), die zur „Besserung“, zur ,Formung des Ich“ beitrügen (Hadot 2002, S. 61):

Im geschriebenen Werk spiegelt sich [...] eine pädagogische, psychagogische, methodologische Zielsetzung. Eigentlich besteht das philosophische Werk, obwohl Geschriebenes ein Monolog ist, immer implizit in einem Dialog; der Bezug auf einen möglichen Gesprächspartner ist immer vorhanden (Hadot 2002, S. 66).

\section{Hebamme und Zitterrochen}

Die ungebrochene Faszination, die „das berühmte ,maieutische“ Kunststück“ (Stenzel 1928, S. 148) des Menon in den letzten drei Jahrhunderten auf die Pädagogik ausgeübt hat, ließ eine weitere mögliche Verbindung zwischen Hebamme und Zitterrochen fast in Vergessenheit geraten. So besteht in „der alten, wundersüchtigen Zeit“ eine medizinische ,Beziehung' zwischen Geburtshelferinnen und Zitterrochen, da die ,so gewaltig und unerklärlich wirkende[n] Thiere in der sogenannten Arzneikunde“" verwendet wurden:

Dem Genusse des schlechten Fleisches wurden die großartigsten Wirkungen zugeschrieben, von den damaligen Ärzten überhaupt über die Heilkräfte dieser Fische Dinge erzählt, welche nur in den Berichten der Homöopathen und anderer Quacksalber unserer Tage gleichwertige Seitenstücke gefunden haben ([Brehm] 1879, S. 384).

Um eine Vorstellung der ,großartigsten Wirkungen“ zu haben, auf die Brehms Thierleben vermutlich anspielt, lohnt sich ein Blick in Plinius' Naturkunde: Zitterrochen ,in der Speise“ reinige den Leib, „durch Auflegen“ des Fisches, „den man dann lebend wieder ins Meer zurückgibt“", könne ebenso die Milz geheilt wie ein „Mastdarmvorfall““ wieder in Ordnung gebracht werden. Die Galle eines lebenden Zitterrochens ,,auf die Geschlechtsteile gestrichen“ solle den „Geschlechtstrieb“ hemmen. ${ }^{9}$ Als ,[m]erkwürdig“ stuft Plinius hingegen folgende Rezeptur ein: Ein Zitterrochen, ,wenn der Mond im Zeichen der Waage steht, gefangen und drei Tage unter freiem Himmel aufbewahrt", solle, „sooft er hereingetragen wird, eine leichte Geburt bewirken“(Plinius 1995, S. 93/32.133). 1563 zählt Konrad Gessner in seinem Fischbuoch denn auch als „Arzney von solchen fischen“ unter anderem auf: „Die läbendigen zitterfisch werdend aufgelegt/ denen so alte haupt wee habend“, ,item dem prästen des miltzes/ ursachet auch ein ringe geburt in die stuben getragen“ (Gessner 1563, S. 76). Ob Plinius jedoch tatsächlich ,in die stuben getragen“ meint, daran zweifelt Paul Diepgen in seiner Frauenheilkunde der Alten Welt (1937). Diepgen fasst die Prozedur, ,quotiens inferatur“, anders auf:

[E]s fragt sich, wie man es zu verstehen hat, wenn Plinius sagt, dass das Tier bei einem bestimmten Mondstand gefangen und 3 Tage unter freiem Himmel aufbewahrt werden soll, und was er unter ,inferre' (anwenden oder einnehmen) versteht (Diepgen 1937, S. 178).

Gibt man inferre mit ,anwenden “ oder, einnehmen“ wieder, dann ist unklar, ob es sich bei der „Applikation des Zitterrochens“ um ,die Verwendung der Elektrizität zur Erleichte- 
rung der Geburt“ oder um „eine innere Darreichung des Tieres“ handelt. Die ,elektrische“ „äußere Anwendung“ wäre durchaus denkbar, hat man doch in der Antike „bei Kopfschmerzen elektrische Schläge durch das lebende Tier empfohlen, wie aus den Compositiones medicamentorum des Scribonius Largus (um 50 n. Chr.) hervorgeht“" (Diepgen 1937, S. 178). Nach „Galen's und Anderer Zeugnis“ preisen „die Alten“, wie W. Keserstein in „Die elektrischen Fische“ 1859 darlegt,

die Schläge des Zitterrochens gegen Kopfweh, Podagra und andere Leiden. Bei Podagra soll man nach Scribonius Largus die Beine in's Meer zu einer Torpedo setzen, so dass man ihre Schläge bis in's Knie hinein fühlt (Keserstein 1859, S. 39 f.).

Der Vergleich von Sokrates’ prüfender „Kunst“ mit der einer Hebamme (Platon 2004c, S. 42/150a-c), die Beschreibung des Philosophen als Zitterrochen sowie Sokrates' Präzisierung, er sei ein sich selbst ,narkotisierender" „[Z]itterfisch“, könnten also alle durchaus auch als Hinweise auf die sonderbare Heilkunst des Philosophen aufgefasst werden. Davon auszugehen, dass nicht nur dem Vergleich mit einer Hebamme, sondern auch dem mit einem Zitterrochen eine medizinische Bedeutung zukommt, scheint um so näher zu liegen, als das Vorbild der Philosophie in der Antike die Medizin und nicht etwa die Pädagogik ist: Mindestens seit Platon präsentiert sich die antike Philosophie als ,therapeutisch“ (vgl. Hadot 2002, S. 369). ${ }^{10}$ Wie Werner Jaeger in seinem Kapitel „Die griechische Medizin als Paideia“" betont:

Wir übertreiben nicht, wenn wir sagen, dass die ethische Wissenschaft des Sokrates, die in Platos Dialogen im Mittelpunkt der Auseinandersetzung steht, ohne das Vorbild der Medizin, auf das Sokrates sich beruft, nicht denkbar gewesen wäre (Jaeger 1944, S. 11).

Die Bedeutung des Vorbilds lässt sich nicht zuletzt auch am Wortschatz erkennen: Bei Platon sind zahlreiche ,terminologische Übereinstimmung[en]“ mit der ,,medizinischen Literatur" feststellbar (Hoffmann 1963, S. 1072). Und natürlich wird die Medizin bei Platon auch häufig in Vergleichen herangezogen. So fordert etwa im Gorgias Sokrates Polos auf, sich ihm ,ruhig der Untersuchung an[zuvertrauen] wie einem Arzt“ (Platon 2004a, S. 76/475e). Im Dialog Sophistes wird die „dialektische Kunst“ der Prüfung von Argumenten sogar als Laxativum beschrieben. Sie helfe bei der inneren „Reinigung“

gerade so, wie die Ärzte bei leiblichen Kuren. Diese sind nämlich überzeugt, der Körper könne erst dann die ihm dargebotene Nahrung mit Nutzen in sich aufnehmen, wenn man die Hindernisse in ihm weggeräumt habe. So sind denn auch jene [welche die dialektische Prüfung vornehmen] rücksichtlich der Seele der Überzeugung, dass sie nicht eher Nutzen haben werde von den ihr dargereichten Wissensschätzen, als bis der Widerlegende seinen Gegner zur Scham vor sich selbst gebracht, und so die der Belehrung hinderlichen Wahnvorstellungen weggeräumt, ihn gereinigt und mit der Überzeugung erfüllt habe, nur das zu wissen, was er wirklich weiß, sonst aber nichts (Platon 2004d, S. 53 f./230b-d).

Diese „Reinigung“ scheint zumindest bei Platon nicht ohne sophistische Tricks und Kniffe zu funktionieren. In „Die sokratische Prüfung: Beobachtungen an platonischen Frühdialogen“ (1967) hebt auch Horst Rumpf hervor, dass Sokrates sich als Arzt betä- 
tige. Die zum Teil widersinnigen und hinterhältigen Schlüsse des Philosophen seien Teil der medizinischen Untersuchung, bei der Sokrates seinen Gesprächspartnern die Brust abklopfe: Wenn die Teilnehmer der Unterredungen wirklich „,besonnen“ wären, fiele es ihnen „leicht, die schwachen Argumente“ des Philosophen ,zurückzuweisen“. Je „dürftiger und grotesker die Widerlegungen durch Sokrates“ (Rumpf 1971, S. 212 f.), umso ausgeprägter müssten die „Wahnvorstellungen“ sein.

\section{Anmerkungen}

1 Künftig verweist die Sigle $m$ in diesem Beitrag auf Platons Menon (Platon 2004b). - Zu Sokrates',negativer' Mäeutik und ihrer Stellung in der Pädagogik vgl. Bühler (2009).

2 Vor Robert Fulton (1812) experimentiert schon sein Landsmann David Bushnell mit Seeminen, wobei er zumindest einmal, 1778, dafür auch den Ausdruck, torpedo 'verwendet zu haben scheint, vgl. Hutcheon (1981), S. 51 f., 84.

3 Vgl. Gray (1975), S. 52 ff., 65, 68, 101.

4 Im Französischen z.B. bedeutet torpille ebenfalls sowohl ,Zitterrochen“ als auch ,Torpedo“, der Ausdruck findet für verschiedene Arten von Seeminen nach 1812 als Übersetzung von Fultons torpedo im Französischen Verbreitung, vgl. [Larousse] (2005).

5 Zur Verbindung von „Hebammendienst“ und Menon vgl. z. B. Buck (1996, S. 208); Burkard u. Weiß (2008, S. 19); Cassau (1882, S. 15); Eppinger (1994, S. 109); Freudenthal (1977, S. 97 f.); Martens (2007, S.76); Petzelt (2005, S. 63 ff.); Prange (1986, S.29); (2008, S.22); Rekus (1999, S. 158 f.); Struve u. Voigt (1988, S. 259); Zöllner (2006, S. 272).

6 Die beiden Abbildungen sind Johann Friedrich Christoph Gräffes Sokratik entnommen, vgl. Gräffe (1791, S. 88, 98). - Weil Gräffe ,dieses Stück aus dem Plato so sehr gefiel“, macht er „bei einem jungen Bauer, der nichts von Mathematik versteht, sondern nur gesunden Menschenverstand besitzt, damit die Probe", die angeblich gelingt (Gräffe 1791, S. 100).

7 Vgl. z.B. Badertscher u. Grunder (1996, S. 9 f.); Benner (2003, S. 97 ff.); Benner u. English (2004, S. 411 ff.); Böhm (1892, S. 96 ff.); Burkard u. Weiß (2008, S. 18 f.); Eppinger (1994, S. 109 ff.); Friedrich (1891, S. 229 ff.); Kapp (1833, S. 144 ff.); Kayser (1890, S. 35 ff.); Kellner (1862, S. 51 ff.); Lindner (1884, S. 858 f.); Menck (1999, S. 214 ff.); Schulz (1849, S. 633 ff.); Schumann (1881, S. 34 ff.); Stenzel (1928, S. 148 ff.); Struve u. Voigt (1988, S. 261 ff., 274); Wagenschein (1962, S. 45 f.); Zöllner (2006, S. 280). - Die ,Mathematikstunde' wird auch ohne die Hilfe von Grafiken erörtert, vgl. z.B. Benner u. Kemper (2003, S.36 ff.); Berg u. Schulze (1997); Cassau (1882, S. 15 f.); Copei (1969, S. 19 ff., 42); Draken (2002); Freudenthal (1977, S. 97 ff.); Green (1987); Günzler (1967, S. 890 ff.); Kamuf (1989, S. 104, 106 f.); Ladenthin (2007, S. 45 ff.); Martens (2007, S. 75 ff.); Meyer-Drawe (2008, S. 202 ff.); Müllges (1986, S. 17 ff.); Pleines (1985, S. 199 ff.); Prange (1973, 1986, S. 26 ff.); Reble (1992, S. 16 ff.); Vrettos (1985, S. 41, $88 \mathrm{ff} ., 100 \mathrm{ff}$.).

8 Die Kritik der platonischen „Fehlschlüsse“ ist nicht neu, z. B. enthält Christoph Martin Wielands Briefroman Aristipp und einige seiner Zeitgenossen ebenfalls eine Reihe „Antiplatonische[r] Briefe“, so eine ausführliche Kritik des Staats. Auch sonst werden in den Briefen oft die ,platten langweiligen Scherz[e]“, die „,blossen Wortspiele“, die „,dialektische Spitzfindigkeit", die ,filosofische[] Mystagogie“ von „Platons sofistisierende[m] Sokrates“ hervorgehoben (Wieland 1988, S. 682, 683, 685, 724, 807, 816).

9 Plinius (1995, S. 69/32.94, 73/32.102, 75/32.105, 95 f./32.139). 
10 Als Arznei ist die Philosophie bei Platon gerade auch deswegen vonnöten, weil die Erziehung versagt; vgl. Foucault (2001, S. 44, 74, 84).

\section{Literatur}

Badertscher, H., \& Grunder, H. U. (1996). Vorwort der Herausgeber. In A. Hollenstein (Hrsg.), Schreibanlässe im Mathematikunterricht. Eine Unterrichtsform für den anwendungsorientierten Mathematikunterricht auf der Sekundarstufe (S.9-12). Bern: Haupt.

Benner, D. (2003). Kritik und Negativität. Ein Versuch zur Pluralisierung von Kritik in Erziehung, Pädagogik und Erziehungswissenschaft. In D. Benner, M. Borrelli, F. Heyting, \& C. Winch (Hrsg.), Kritik in der Pädagogik. Versuche über das Kritische in Erziehung und Erziehungswissenschaft (Zeitschrift für Pädagogik, Beiheft 46, S.96-110). Weinheim: Beltz.

Benner, D., \& English, A. (2004). Critique and negativity: Towards the pluralisation of critique in educational practice, theory and research. Journal of Philosophy of Education, 38, 409-428.

Benner, D., \& Kemper, H. (2003). Theorie und Geschichte der Reformpädagogik. Bd 1: Die pädagogische Bewegung von der Aufklärung bis zum Neuhumanismus (2.Aufl.). Weinheim: Beltz.

Berg, H. C., \& Schulze, T. (1997). Editorial. In H. C. Berg \& T. Schulze (Hrsg.), Lehrkunstwerkstatt I. Didaktik in Unterrichtsexempeln (S. 83-84). Neuwied: Luchterhand.

Bertuch, F. J. (1790). Bilderbuch für Kinder enthaltend eine angenehme Sammlung von Thieren, Pflanzen, Blumen, Früchten, Mineralien, Trachten und allerhand andern unterrichtenden Gegenständen aus dem Reiche der Natur, der Künste und Wissenschaften; alle nach den besten Originalen gewählt, gestochen, und mit einer kurzen wissenschaftlichen, und den Verstandes-Kräften eines Kindes angemessenen Erklärung begleitet (1.Bd.). Weimar: Industrie-Comptoir.

Böhm, J. (1892). Die Geschichte der Pädagogik mit Charakterbildern hervorragender Pädagogen und Zeiten. 1.Bd: Die Geschichte der Pädagogik vor und nach Christus bis auf Montaigne (2.Aufl.). Nürnberg: Korn.

[Brehm] (1879). [Eintrag] ,Zitterrochen“. In Brehms Tierleben. Allgemeine Kunde des Thierreichs. 3. Abteilung, 2.Bd.: Kriechthiere, Lurche und Fische (2.Aufl., S. 382-385). Leipzig: Verlag des Bibliographischen Instituts.

[Brockhaus] (2001). [Eintrag], Zitterrochen‘. In Brockhaus. Die Enzyklopädie in 24 Bänden. (20.Aufl., 24. Bd., S. 604). Mannheim: Brockhaus.

Buck, G. (1996). Das Lehrgespräch. In K. Stierle \& R. Warning (Hrsg.), Das Gespräch (Poetik und Hermeneutik, 11. Band, 2.Aufl., S. 191-210). München: Fink.

Bühler, P. (2009). Sokrates' Hebammenkünste und die Pädagogik. Vierteljahrsschrift für wissenschaftliche Pädagogik, 89, 285-301.

Burkard, F.-P., \& Weiß, A. (2008). dtv-Atlas Pädagogik. München: Deutscher Taschenbuch Verlag.

Cassau, K. (1882). Die Pädagogik der Alten. Charakterbilder und Skizzen. Leipzig: Dürr.

Copei, F. (1969). Der fruchtbare Moment im Bildungsprozess (9.Aufl.). Heidelberg: Quelle \& Meyer.

Diepgen, P. (1937). Die Frauenheilkunde der Alten Welt. München: Bergmann.

Draken, K. (2002). Mit Sokrates zur modernen Diskurstheorie. Ein Unterrichtsreihe für die Jahrgangstufe 11. Ethik \& Unterricht, 12(1), 32-39.

Eckert, W. (1911). Dialektischer Scherz in den frühen Gesprächen Platons. Nürnberg: Sebald.

Engel, J. J. (1805). Versuch einer Methode, die Vernunftlehre aus Platonischen Dialogen zu entwickeln. (S. 1-200). In J. J. Engel, Schriften. 9.1 Band. Berlin: Mylius.

Eppinger, F. (1994). Praxis Lehrerfortbildung: Platonischer Dialog und gruppenpädagogisches Konzept. Pädagogisches Forum, 7(3), 107-116. 
Foucault, M. (2001). L’herméneutique du sujet. Cours au Collège de France 1981-1982. Paris: Gallimard, Seuil.

Friedrich, G. (1891). Sokrates und seine Lehrweise mit Rücksichtnahme auf den Platonischen Menon. Deutsche Blätter für erziehenden Unterricht, 18(28), 221-224, (29), 229-233. www.bbf.dipf.de/cgi-shl/digibert.pl?id=BBF0772149 und www.bbf.dipf.de/cgi-shl/digibert. pl?id=BBF0772169. Zugegriffen: 11. März 2010.

Freudenthal, H. (1977). Die sokratische Methode. In H. Freudenthal, Mathematik als pädagogische Aufgabe (Bd. 1, 2. Aufl., S.97-105). Stuttgart: Klett.

Fulton, R. (1812). De la machine infernale maritime, ou de la tactique offensive et défensive de la Torpille. Description de cette Machine, et Expérience faites en Angleterre et aux Etats-Unis, sur la manière d'en faire usage. Paris: Magimel.

Gessner, K. (1563). Fischbuoch. Das ist ein kurtze/doch vollkommne beschreybung aller Fischen so in dem Meer und süssen wasseren/Seen/Flüssen/oder anderen Bächen ir wonung habend/[...]. Zürich: Froschower.

Gräffe, J. F. C. (1791). Neustes Katechetisches Magazin zur Beförderung des katechetischen Studiums. 2. Bd.: Die Sokratik nach ihrer ursprünglichen Beschaffenheit in katechetischer Rücksicht betrachtet. Göttingen: Vandenhoeck \& Ruprecht.

Gray, E. (1975). The devil's device. The story of Robert Whitehead, Inventor of the torpedo. London: Seeley.

Green, J. L. (1987). Meno's motivation: The foundations of learning. The Educational Forum, 51 (2), 151-165.

Günzler, C. (1967). Platons Begriff des Lernens und das Problem der Sachlichkeit. Pädagogische Rundschau, 21, 887-904.

Hadot, P. (2002). Exercices spirituels et philosophie antique. Nouvelle édition revue et augmentée. Paris: Albin Michel.

Hamann, J. G. (1959). Sokratische Denkwürdigkeiten (Johann Georg Hamanns Hauptschriften erklärt, 2. Bd.). Gütersloh: Mohn.

Hoffmann, E. (1963). Platon und die Medizin. In E. Zeller, Die Philosophie der Griechen in ihrer geschichtlichen Entwicklung. Bd.2.1: Sokrates und die Sokratiker. Plato und die alte Akademie (6. Aufl., S. 1070-1086). Darmstadt: Wissenschaftliche Buchgesellschaft.

Hutcheon, W. (1981). Robert Fulton. Pioneer of Undersea Warfare. Annapolis: Naval Institute Press.

Jaeger, W. (1944). Paideia. Die Formung des griechischen Menschen (2. Bd.). Berlin: de Gruyter.

Kamuf, U. (1989). Zur Sokratischen Methode. In I. Heydorn \& B. Schmidt (Hrsg.), Traditio lampadis. Das Versöhnende dem Zerstörenden abtrotzen. Festgabe für Gernot Koneffke (S.103113). Liechtenstein: Topos.

Kapp, A. (1833). Platon's Erziehungslehre, als Pädagogik für die Einzelnen und als Staatspädagogik. Oder dessen praktische Philosophie. Aus den Quellen dargestellt. Minden: Essmann.

Kayser, J. (1890). Kehreins Überblick der Geschichte der Erziehung und des Unterrichts für die Zöglinge der Lehrerseminare und zur Vorbereitung auf die in den Allg. Bestimmungen angeordneten Prüfungen (9.Aufl.). Paderborn: Schöningh.

Kellner, L. (1862). Skizzen und Bilder aus der Erziehungsgeschichte. Mit besonderer Rücksicht auf das Volksschulwesen, für Lehrer, deren Bildner und Leiter (1.Bd.). Essen: Bädeker.

Keserstein, W. (1859). Die elektrischen Fische. Westermann's Illustrirte Deutsche Monatshefte, 31(2. Abteilung), 33-48.

Kierkegaard, S. (1961). Über den Begriff der Ironie. Mit ständiger Rücksicht auf Sokrates (Gesammelte Werke: 31. Abteilung). Düsseldorf: Diederichs.

Kluge, F. (1975). [Eintrag] ,Torpedo‘. In F. Kluge (Hrsg.), Etymologisches Wörterbuch der deutschen Sprache (21.Aufl., S. 784). Berlin: de Gruyter.

Ladenthin, V. (Hrsg.). (2007). Philosophie der Bildung. Eine Zeitreise von den Vorsokratikern bis zur Postmoderne. Bonn: DenkMal. 
[Larousse] (2005). [Eintrag], torpille‘. In J. Dubois, H. Mitterand \& A. Dauzat (Hrsg.), Grand Dictionnaire étymologique et historique français (S. 1002). Paris: Larousse.

Lindner, G. A. (1884). [Eintrag] ,Sokrates'. In G. A. Lindner (Hrsg.), Encyklopädisches Handbuch der Erziehungskunde mit besonderer Berücksichtigung des Volksschulwesens (S. 854-860). Wien: Pichler.

Martens, E. (2007). Sokrates im Schulunterricht. In B. Reis (Hrsg.), Zwischen PISA und Athen - Antike Philosophie im Schulunterricht (S. 73-88). Göttingen: V \& R unipress.

Menck, P. (1999). Geschichte der Erziehung (2.Aufl.). Donauwörth: Auer.

Mendelssohn, M. (1760). [Unter Pseudonym veröffentlichte Rezension zu:] Johann Georg Hamann Sokratische Denkwürdigkeiten. Briefe, die neueste Literatur betreffend, 6(113. Brief), 19. Juni 1760, 385-400. www.ub.uni-bielefeld.de/diglib/aufklaerung/index.htm. Zugegriffen: 11. Feb. 2010.

[Meyer] (1897). [Eintrag] ,Sokrates‘. In Meyers Konversations-Lexikon. Ein Nachschlagewerk des allgemeinen Wissens. (5.Aufl., 16. Bd., S. 69-70). Leipzig: Bibliographisches Institut.

Meyer-Drawe, K. (2008). Diskurse des Lernens. München: Fink.

Montaigne, M. de (1992). Essais [Versuche] nebst des Verfassers Leben nach der Ausgabe von Pierre Coste ins Deutsche übersetzt von Johann Daniel Tietz. Zweeter Theil. Zürich: Diogenes.

Mosheim, J. L. (1737). Sitten-Lehre der Heiligen Schrift (1.Bd., 2.Aufl.). Helmstädt: Weygand.

Moszkowski, A. (1917). Sokrates der Idiot. Eine respektlose Studie. Berlin: Eysler.

Müllges, U. (1986). Quellen zur historischen Didaktik und Methodik. Mannheim: Bibliographisches Institut.

[OED]. (1989). [Eintrag] ,torpedo‘. In J. A. Simpson \& E. S. C. Weiner (Hrsg.), The Oxford english dictionary (2.Aufl., 18. Bd., S. 270-271). Oxford: Clarendon.

Petzelt, A. (2005). Platon als Erzieher. In K. G. Pöppel (Hrsg.), Problemgeschichte der Pädagogik. Grundzüge und Beispiele. Eine Einführung (S.13-125). Hildesheim: Olms.

Platon (2004a). Gorgias. In Platon (Hrsg.), Sämtliche Dialoge (1. Bd., S. 1-184). Übersetzt und erläutert von O. Apelt. Hamburg: Meiner.

Platon (2004b). Menon. In Platon (Hrsg.), Sämtliche Dialoge (2. Bd., S. 1-91). Übersetzt und erläutert von Otto Apelt. Hamburg: Meiner.

Platon (2004c). Theätet. In Platon (Hrsg.), Sämtliche Dialoge (4. Bd., S.1-195). Übersetzt und erläutert von Otto Apelt, Hamburg: Meiner.

Platon (2004d). Sophistes. In Platon (Hrsg.), Sämtliche Dialoge (6. Bd., S. 1-156). Übersetzt und erläutert von Otto Apelt, Hamburg: Meiner.

Pleines, J.-E. (1985). Ist Tugend lehrbar? Im Gedenken an Günther Buck. Vierteljahrsschrift für wissenschaftliche Pädagogik, 61, 197-212.

Plinius Secundus, G. (1979). Naturkunde. Lateinisch - deutsch. 9. Buch: Zoologie: Wassertiere. München: Heimeran.

Plinius Secundus, G. (1995). Naturkunde. Lateinisch-deutsch. 32. Buch: Medizin und Pharmakologie: Heilmittel aus dem Wasser. München: Heimeran.

Prange, K. (1973). Platos Lehre vom Lernen im ,Menon` und das Problem des Allgemeinen. Pädagogische Rundschau, 27(10), 685-700.

Prange, K. (1986). Bauformen des Unterrichts. Eine Didaktik für Lehrer (2.Aufl.). Bad Heilbrunn: Klinkhardt.

Prange, K. (2008). Schlüsselwerke der Pädagogik. 1. Bd.: Von Plato bis Hegel. Stuttgart: Kohlhammer.

Reble, A. (1992). Geschichte der Pädagogik. Dokumentationsband (2.Aufl.). Stuttgart: Klett-Cotta.

Rekus, J. (1999). Das Fragen im Prozess der Bildung. Grundlegende Überlegungen und praktische Orientierungen. In T. Bürklin \& C. von Wolzogen (Hrsg.), Gegenwendiges Denken. „,Metaphysische "Fragen zwischen Philosophie und Pädagogik (S. 156-166). Hildesheim: Olms. 
Rumpf, H. (1971). Die sokratische Prüfung. Beobachtungen an platonischen Frühdialogen. In H. Rumpf, Scheinklarheiten. Sondierungen von Schule und Unterrichtsforschung (S. 204-219). Braunschweig: Westermann.

Schlegel, F. (1975). Lessings Gedanken und Meinungen aus dessen Schriften zusammengestellt und erläutert. In F. Schlegel, Charakteristiken und Kritiken II (1802-1829) (Kritische FriedrichSchlegel-Ausgabe, Bd.3.1, S. 46-103). München: Schöningh, Thomas.

Schulz, O. (1849). Die sokratische Lehrmethode. Schulblatt für die Provinz Brandenburg, 14(11/12), 618-648. www.bbf.dipf.de/cgi-shl/digibert.pl?id=BBF0823902. Zugegriffen: 11. Feb. 2010.

Schumann, J. C. G. (1881). Lehrbuch der Pädagogik. 1. Bd.: Einleitung und Geschichte der Pädagogik mit Musterstücken aus den pädagogischen Meisterwerken der verschiedenen Zeiten (6. Aufl.). Hannover: Meyer.

Stenzel, J. (1928). Platon der Erzieher. Leipzig: Meiner.

Struve, R., \& Voigt, J. (1988). Die Unterrichtsszenen im Menon-Dialog. Analyse und Kritik auf dem Hintergrund von Interaktionsanalysen des heutigen Mathematikunterrichts. Journal für Mathematik-Didaktik, 9(4), 259-285.

Terhart, E. (2009). Didaktik. Eine Einführung. Stuttgart: Reclam.

Vrettos, J. (1985). Lehrer-Schüler-Interaktion bei Platon. Die pädagogische Bedeutung von Eros und Dialog. Frankfurt a. M., Bem, New York: Peter Lang.

Wagenschein, M. (1962). Der antike Beweis für die Irrationalität der Quadratwurzel aus 2. Der Mathematikunterricht, 8(4), 39-54.

Walther, F. R. (1780). Das Charakteristische der Sokratischen Lehrart. Archiv für die ausübende Erziehungskunst, 7, 1-40.

Weierstraß, K. (1903). Über die Sokratische Lehrmethode und deren Anwendbarkeit beim Schulunterrichte. In K. Weierstraß, Mathematische Werke (3.Bd., S.315-329). Berlin: Mayer \& Müller.

Wetzel, J. C. F. (1801). Sokratik. Womit zur Schulprüfung auf den 23. April 1801 einen Hochedlen Magistrat als Patron, ein Hochzubeehrendes Ephorat, und alle Gönner und Freunde unsers Lyceums ehrerbietigst einladet. Prenzlau: Ragoczy.

Wieland, C. M. (1988). Aristipp und einige seiner Zeitgenossen (Werke in zwölf Bänden, 4. Bd.). Frankfurt a. M.: Deutscher Klassiker Verlag.

Zöllner, D. (2006). Individuelles und kulturelles Lernen: erkenntnistheoretische Anmerkungen zum Lernbegriff. Pädagogische Rundschau, 60, 271-295. 